\title{
Morphological Characteristics of Liver Abscesses According its Etiology
}

\author{
Características Morfológicas de los Abscesos Hepáticos Según su Etiología
}

\author{
Sergio Castillo ${ }^{1,2} \&$ Carlos Manterola ${ }^{1,3,4}$
}

CASTILLO, S. \& MANTEROLA, C. Morphological characteristics of liver abscesses according its etiology. Int. J. Morphol., 38(2):406414, 2020.

SUMMARY: Although infrequent in the diagnostics areas, liver abscesses are of significant relevance, which is related to the high morbidity and mortality figures they can cause if they are not detected and treated in time. Although multiple operational classifications are described, the one most recognized for its usefulness is that related to its causative agent (pyogenic or bacterial infection, hydatid origin or secondary to infected hydatidosis, and amoebic or by invasive amebiasis). The objective of this article was to generate a study report regarding the morphological characteristics of liver abscesses, characterizing them according to their etiology, as well as describing their study and the latest recommended treatments.

KEY WORDS: "Liver Abscess"[Mesh]; “Echinococcosis, Hepatic”[Mesh]; "“Liver Abscess, Pyogenic"[Mesh]; "Liver Abscess, Amebic”[Mesh]; Hydatidosis, Hepatic.

\section{INTRODUCTION}

Hepatic abscess (HA) is a localized process of encapsulated suppurative material. Although rare, it is associated with morbidity and mortality figures of 2-12\%. These rates increase when adequate diagnosis and treatment are delayed, namely within the context of a secondary sepsis (Abbas et al., 2014; González-Alcaide et al., 2017; Akhondi \& Sabih, 2019).

There are various forms in which HA may be classified: According to its anatomical location (hemi-liver / segment (s) involvement, depth of the lesion), number of injuries (single or multiple), routes of infection (portal, biliary, contiguous), type of treatment proposed (antimicrobials, guided puncture drainage, surgery) and by the causative agent. Most infections are usually caused by bacteria or amoebas (mainly Entamoeba histolytica), although they may also be secondary to infections caused by other parasites (generally of the genus Equinococcus) and fungi (González-Alcaide et al.; Shi et al., 2018).

It is therefore, important to consider specific populations in which the incidence, prevalence and risk of HA occurrence increases, when compared to the general population. Immunocompromised subjects, such as those with infective endocarditis, colorectal neoplasia, and intravenous drug users (regardless of the underlying cause) may frequently develop a pyogenic abscess (PA) (Abbas et al.; Akhondi \& Sabih). Women with prior history of pelvic inflammatory disease, may present with Neisseria gonorrhea, Chlamydia trachomatis or Mycobacterium tuberculosis infection, related to Fitz-Hughes-Curtis complications. Amebic hepatic abscess (AHA) is more common in populations without access to clean drinking water, (González-Alcaide et al.); HA caused by Echinococcus granulosus infection, its complications and progression also known as hydatid hepatic abscess (HHA), has been confirmed in endemic regions (Akhondi \& Sabih).

The aim of this manuscripr was to develop a documented study regarding the morphological characteristics of the different types of AH. For classification purposes these will be classified as PHA, AHA and HHA.

\footnotetext{
${ }^{1}$ Doctoral Program in Medical Sciences, Universidad de La Frontera, Temuco, Chile.

${ }^{2}$ Clínica RedSalud Mayor Temuco, Temuco, Chile.

${ }^{3}$ Department of Surgery, Universidad de La Frontera, Temuco, Chile.

${ }^{4}$ Center of Morphological and Surgical Studies (CEMyQ), Universidad de La Frontera, Temuco, Chile.

FUNDING: Partially funded by project DI16-0119, Universidad de La Frontera, Temuco, Chile.
} 


\section{Pyogenic hepatic abscess}

PHA is an infrequent pathology, although when it is not diagnosed and treated timely, results in a range of mortality rates close to $100 \%$. Incidence varies between 2 and 15 cases per 105 inhabitants /year. It is reported more frequently in countries with poor sanitary conditions and often affects 40 to 70 -year-old individuals; it is also twice as likely in men than in women. Mortality varies between $15 \%$ and $20 \%$, herein the following factors indicate poor prognosis: Development of subsequent sepsis; existence of multiple abscesses; polymicrobial infection; resistance to antibiotics; being over 70 years of age; association with neoplasia and immunosuppression (Pinilla et al., 2003).

The pathogenic mechanism in these cases is complex and multiform. Abscess may be due to acute cholangitis secondary to biliary diseases ("cholangitic abscesses"). Estimates indicate these to be the cause of $30 \%$ of cases (Shi et al.). Abdominal infectious processes which may or not, be associated with portal vein thrombosis ("pileflebitic abscesses") are responsible for approximately $20 \%$ of cases (Piqueras et al., 2016). In addition, secondary bacteremia of infections with hepatic dissemination throughout the hepatic artery ("hematogenous abscesses"), non-penetrating trauma and wounds with hematoma infection, direct or extended contact from an adjacent infection and unrelated to the bile ducts. Further causes include ruptured bowel by accidental ingestion of foreign bodies, and treatment procedures of malignant liver lesions. Occasionally, when etiology cannot be identified, the term "cryptogenetic abscesses" is used. Estimates indicate that cholangitic and cryptogenetic HA are the most frequently reported (Longworth \& Han, 2015) (Fig. 1).

Pyogenic microorganisms that spread to the liver through the hepatic portal are permanently eliminated by the hepatic reticuloendothelial system, thereby preventing their proliferation and systemic circulation. This process however, may be altered under primary or acquired immunodeficiency conditions and promote HA development. Consequently, PHA is more frequent in immunosuppressed patients (diabetic, transplanted, neoplasm carriers, patients on immunosuppressant treatment, etc.) (Mischnik et al., 2017).

Clinical manifestations depend on its pathogenic mechanism. Classic symptoms consist of pain in the right hypochondrium (89-100\%) with or without hepatomegaly (51-92\%). Symptoms are also associated with fever (67$100 \%)$, and chills (33-88\%). The presence of jaundice (23$43 \%)$ is predominantly associated with the existence of concomitant biliary disease (Longworth \& Han).
In more than $90 \%$ of cases germ identification is achieved by abscess content culture, and blood culture in over $50 \%$ of cases. More than half of PHAs are reportedly polymicrobial, germs responsible for the disease are related to the primary infection site. Enterobacteria, particularly E. coli and K. pneumonie; Microaerophilic streptococci and intestinal anaerobes, such as Bacteroides spp, have been isolated in over $90 \%$ of cases (Mischnik et al.).

Findings observed in laboratory tests of patients with PHA, such as leukocytosis, elevated levels of alkaline phosphatase and bilirubin, are nonspecific and characteristic of systemic bacterial infection (Piqueras et al.).

PHA diagnosis is determined by imaging exams such as ultrasonography (US), computed tomography (CT), magnetic resonance imaging (MRI), and nuclear medicine (NM, by scintigraphy) and analyses presenting lesion topography and morphology. These may also be useful for an eventual image-guided puncture aspiration for microbiological study, and percutaneous drainage as a therapeutic alternative (Pinilla et al.; Lardière-Deguelte et al., 2015; Mischnik et al.).

\section{Morphological characteristics:}

Pathological anatomy: PHA macroscopic features are either single or multiple cavities, filled (s) with yellowish, often described as 'anchovy paste', foul-smelling material, corresponding to necrotic tissue which may, or may not be surrounded by a fibrous capsule (Fig. 2). The microscopic study allows confirmation of the main necrotic site, along with abundant pyocytes and neutrophils, as well as adjacent reactive hepatocytes (Longworth \& Han; Akhondi \& Sabih).

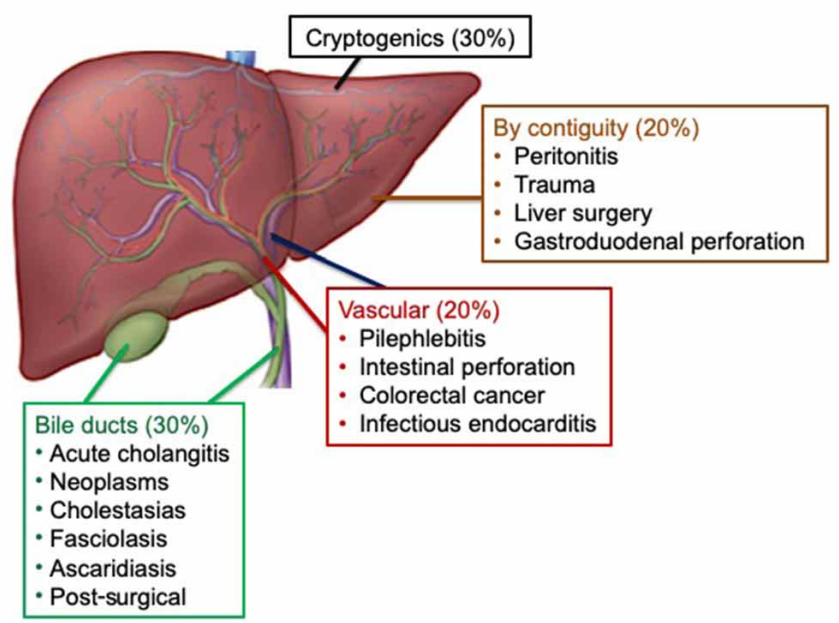

Fig. 1. Dissemination of the pathogen of the pyogenic hepatic abscess. 


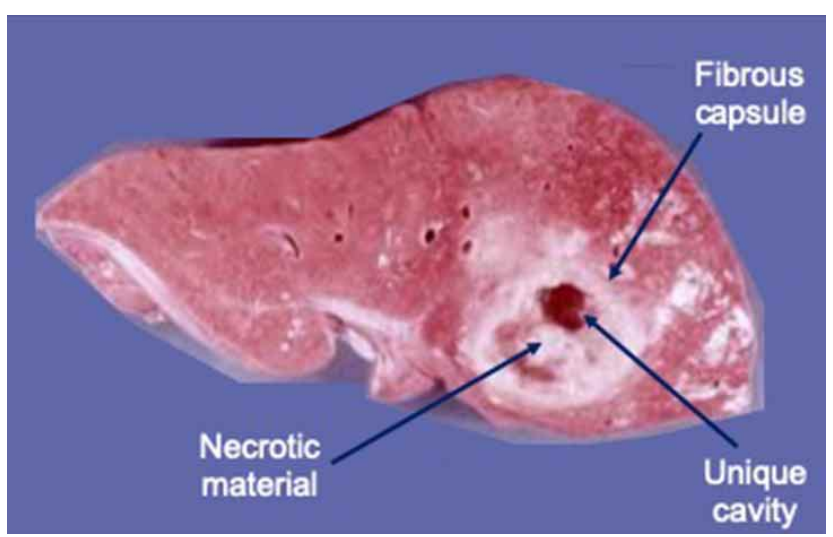

Fig. 2. Section of surgical piece of hepatectomy by PHA. Unique cavity, with fibrous capsule and necrotic material inside.

Images (Tables I and II): In less than $50 \%$ of cases, conventional radiological findings on chest $\mathrm{x}$-ray or simple abdomen $\mathrm{x}$-ray present elevation of the right hemidiaphragm, sub-diaphragmatic or intrahepatic hydro-air levels, pleural effusion, and aerobilia (Zibari et al., 2010) (Tables I and II; Fig. 3A). Highly sensitive (near $100 \%$ ) ultrasound (US) in these cases, usually confirms predominantly hypoechogenic masses which vary in size and appearance. Nonetheless, they may also present as hyperechogenic with inside echoes (Fig. 3B). The existence of gas bubbles is sometimes reported, and Doppler imaging shows the absence of central perfusion. In monomicrobial abscesses resulting from K. pneumoniae, lesions may appear solid and simulate a tumor lesion.

Meanwhile, contrast US may determine reinforcement during the arterial phase, and progressive washout in the late portal phase (necrotic areas are not subject to reinforcement) (Pinilla et al.).

On the other hand, three-phase CT is the study of choice. It allows observation of central images not highlighted on intravenous contrast, accentuated on the inside margin in early and late stage, and hypointense external halo due to edema, which only highlights in the late stage (target sign).

The cluster sign is characteristic of the PHA, consisting of several lesions grouping to form a single multiloculated cavity (Fig. 3C). In its arterial and portal phases, it also allows to define the anatomical relationship to the vessels and program surgical access (Longworth \& Han).

Conversely, NMR provides the following information: T1, heterogeneous central hypointensity; T2, hyperintensity and perilesional edema (35\% of cases); T1 with Gadolinium, thickened capsule reinforcement (may not present in immunocompromised subjects), and multiple septa. In DWI: hyperintensity in the cavity, and in ADC:

Table I. Summary of findings in the various diagnostic image testing, according to type of hepatic abscess.

\begin{tabular}{|c|c|c|c|}
\hline \multicolumn{4}{|c|}{ Image techniques } \\
\hline $\mathbf{U S}$ & TAC & RNM & MN \\
\hline Hypo or hyperechoic Doppler (-) & $\begin{array}{l}\text { Hypo-inten sity, } \\
\text { reinfor ced margin } \\
\text { Cluster sign. }\end{array}$ & $\begin{array}{l}\mathrm{T} 1 \downarrow \mathrm{T} 2 \uparrow \\
\mathrm{DWI} \uparrow \mathrm{ADC} \downarrow \\
\text { Perilesional Edema } \\
\text { T1Gd reinforced }\end{array}$ & "Hot" \\
\hline $\begin{array}{l}\text { Hypoechoic. } \\
\text { Absence of wall echoes }\end{array}$ & $\begin{array}{l}\text { Thick fluid, septum, levels and } \\
\text { edema. }\end{array}$ & $\begin{array}{l}\mathrm{T} 1 \downarrow \text { T2 } \uparrow \text { Perilesional } \\
\text { Edema }\end{array}$ & "Cold" \\
\hline $\begin{array}{l}\text { Variable. } \\
\text { Hypoechoic heterogeneous, with or } \\
\text { without septa }\end{array}$ & Attenuation, variable, septa. & $\begin{array}{l}\mathrm{T} 1 \downarrow \mathrm{T} 2 \uparrow \\
\mathrm{T} 1 \mathrm{Gd} \text { with margin } \\
\text { reinforcement }\end{array}$ & "Cold" \\
\hline
\end{tabular}

Table II. Sum summary of the sensitivity described for the various diagnostic image testing, according to type of hepatic abscess.

\begin{tabular}{lcccc}
\hline & \multicolumn{4}{c}{ Image techniques * } \\
& US & CT & NMR & NM \\
& $(\%)$ & $(\%)$ & $(\%)$ & $(\%)$ \\
\hline PHA & $80-95$ & $95-98$ & 95 & $50-80$ \\
AHA & $80-95$ & 95 & 95 & 50 \\
HHA & 95 & 95 & 95 & No data \\
\hline
\end{tabular}

US: Ultrasonography

CT: Computed tomography

NMR: Nuclear magnetic resonance

NM: Nuclear medicine (gammagraphy or scintigraphy

PHA: Pyogenic hepatic abscess

AHA: Amebic hepatic abscess

HHA: Hydatid hepatic abscess

*: Table data corresponds to that reported in the literature 

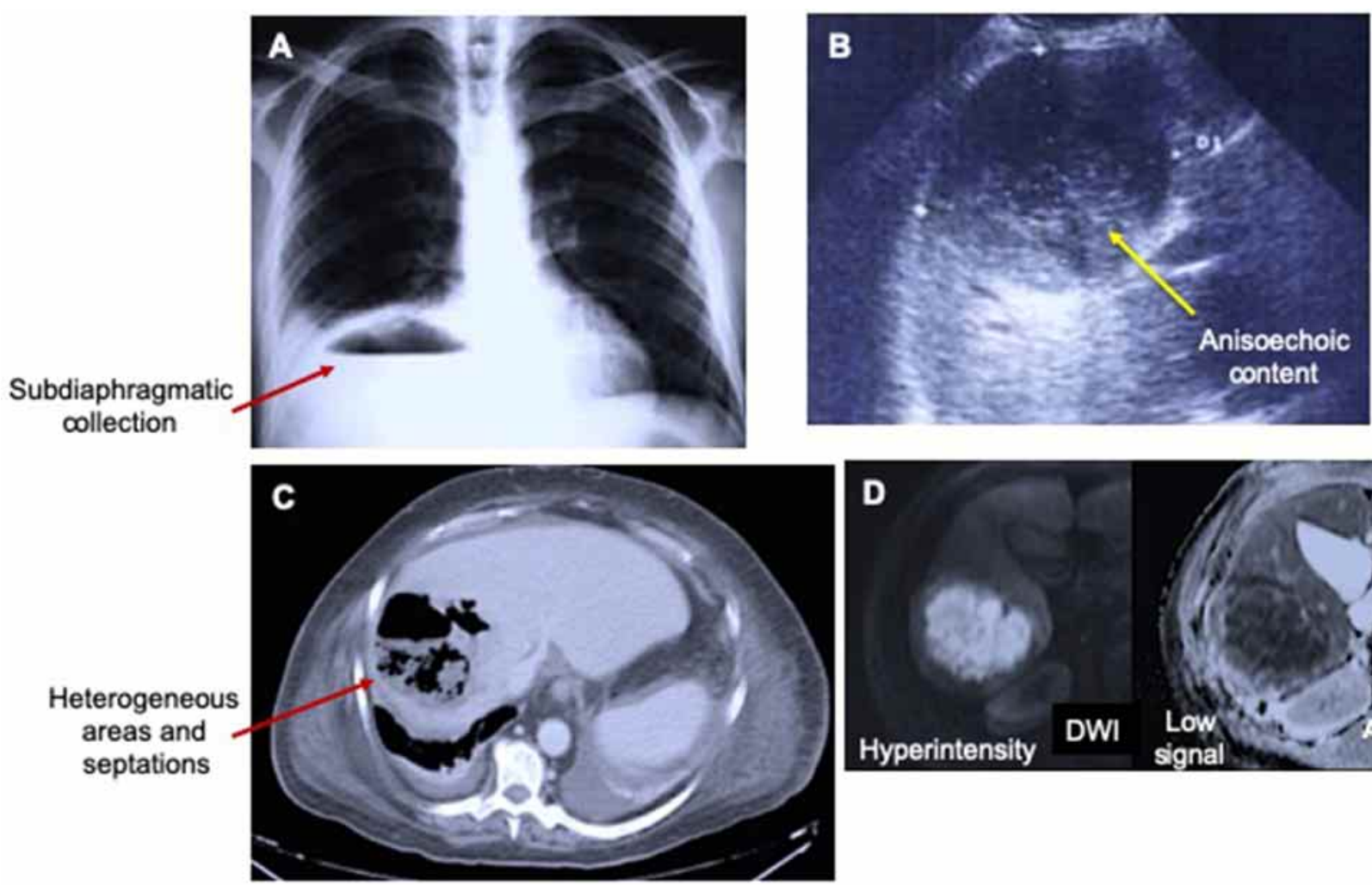

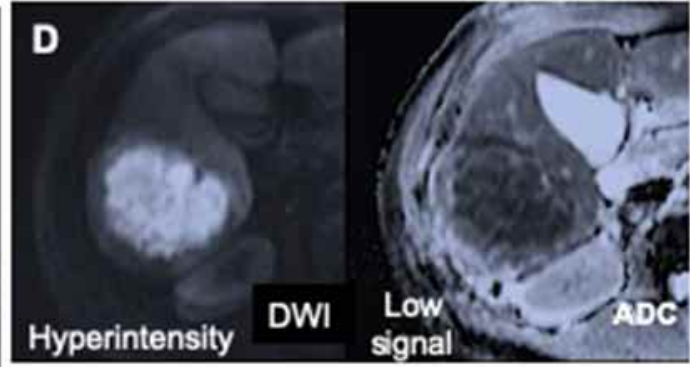

Fig. 3. A: Conventional radiology. In a simple PA chest X-ray, a right subdiaphragmatic hydro-air collection image was observed, cystic in its appearance, corresponding to a PHA. B: PHA US. A predominantly hypoechoic, rounded image with an isoechoic content can be seen. C: PHA with contrast CT scan. Heterogeneous areas of hypodensity and partitions are seen in the right hepatic lobule compatible with PHA. D: NMR contrasted with Gadolinium of PHA in the right hepatic lobule. Hyperintensity in DWI, with low signal in ADC.

low signal low intensity in the cavity (Mischnik et al.) (Fig. 3D).

Finally, the use of NM through radioisotope uptake study (Tc-99m, Gallium), allows identification of abscess "hot spots". Nonetheless, when compared with CT and NMR results, it is no longer estimated as the best choice, considering its sensitivity rating of $50 \%$ to $80 \%$ (Zibari et al.).

In PHA, early antibiotic treatment associated with percutaneous drainage, is successful overall in most patients, with a reported 4 to $11 \%$ rate of complications (Lübbert $e t$ al., 2014). Pharmacological treatment options are considered according to the initial focus of infection (known or assumed), as well as the typically known germs. Combination therapy is usually required to achieve an adequate treatment spectrum (Lardière-Deguelte et al.).

Treatment:Antibiotics are generally administered along with a percutaneous drainage catheter, guided by images, or surgical drainage, which is indicated best in cases where percutaneous drainage or antibiotic treatment

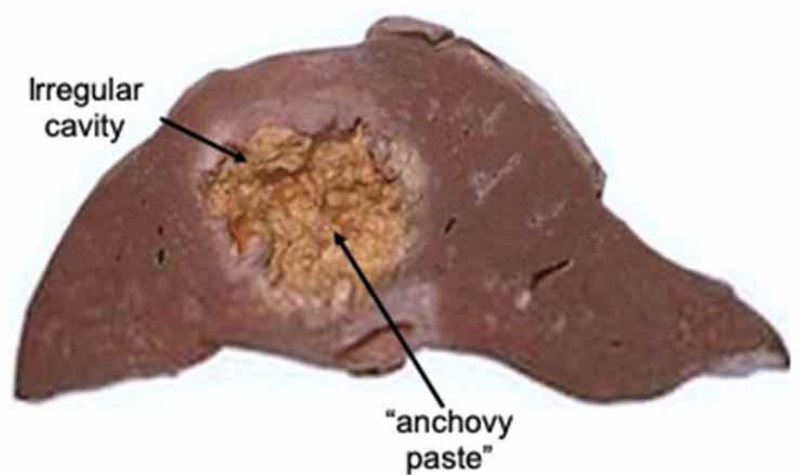

Fig. 4. Section of surgical piece of hepatectomy by PHA. Well circumscribed cavity, with uneven contours, with dark material ("anchovy paste") content.

failed. Access may be via laparoscopic or laparotomic approach, in cases of coexisting abdominal pathology requiring surgery (i.e., Mirizzi syndrome). Also, when percutaneous drainage is not indicated (coagulopathies, existence of ascites, multiple small abscesses, risk of damage to vital organs), and in cases of PHA rupture or hemorrhage. (Lübbert et al.; Lardière-Deguelte et al.; Mischnik et al.). 


\section{Amebian hepatic abscess}

Amebiasis infection by pathogenic amoebas, are typically referred to as Entamoeba histolytica, but which also take into account infections due to E. dispar, E. moshkovski and E. bangladeshi; which make up the collectively referred to Entamoeba Complex (Alam et al., 2014).

Prevalence of this infection is high. Rates of 500 million people infected by parasites are estimated worldwide; with an additional 40 to 50 million infected each year. It is widespread throughout the various regions of the planet (1 $\%$ to $2 \%$ in temperate climate zones, and over $50 \%$ in tropical regions). It is also more common in countries with poor health conditions and access to drinking water, creating endemic areas. This is known as the Entamoeba transmission cycle, the human intestine acting as the reservoir through fecal-oral transmission (Alam et al.). Hence, in western countries, most AHAs are found in migrant populations and travelers from endemic areas. It is more frequent in those individuals remaining in an endemic area, for over 6 weeks. However, the real number of Entamoeba infected subjects who develop AHA, is unknown. AHA has been reported following travels to endemic areas for very brief periods (4 days) (Anesi \& Gluckman, 2015; Lachish et al., 2016).

AHA occurs through liver colonization by pathogenic strains of Entamoeba through the portal circulation, after their trophozoites cross the mucous membrane of the colon and penetrate mesentery venules. Lectin galactose / Nacetyl-D-galactosamine (Gal / GalNAc) adhesion complex, and other adhesins allow the proliferation of pathogenic amoebas, as they also bind to white tissue. Once in the liver, destruction capability is determined by the enzymes with collagenase activity, phosphatases and cysteine proteases that degrade the extracellular matrix and phagocytized tissue. Additionally, via porins (ameboporos A, B and C), which facilitate osmotic cell lysis by forming ion channels. These can also induce apoptosis through the caspase activation process, and anoikis a specific type of apoptosis consisting in the destruction of cell anchoring to the extracellular matrix (Anesi \& Gluckman).

Approximately $60 \%$ of AHAs are unique, showing a preference for the right hemi-liver near the liver dome, which may be due to greater volume and general irrigation. Portal laminar flow in the right liver is supplied mainly by the superior mesenteric vein, while in the left liver the flow is predominantly delivered by the splenic vein (Alam et al.; Lachish et al.).

Although the rate of infection and invasion into the colon is similar in both sexes, AHA is 7 to 12 times more frequent in men, during the 3rd and 5th decades of life, though it can present at any age.

Clinical manifestations are usually acute and nonspecific, with prevalent symptoms in the younger population. These include abdominal pain (approximately in $90 \%$ cases), typically in the upper right quadrant, which may be associated with ipsilateral omalgia. Pain usually increases with cough, deep inspiration, mobility and while resting on the affected side. Fever is reported in $99 \%$ of cases, diarrhea in 30-50\% of cases ( $7 \%$ dysentery), nausea and vomiting (32-85\%), weight loss (33-64\%) and lung involvement (18-26\%). The latter includes cough and chest pain, suggesting possible rupture of the abscess to the pleural cavity (Alam et al.).

Diagnosis is based on the epidemiological background information, clinical scope and images. Additionally, specific serological tests aimed at isolating the parasite may be used, such as enzyme immunoassay (EIA), using antibodies binding the antigen, and enzyme, usually a chromogen or fluorogen to detect the antigen (Alam et al.). This method is readily available, easy to use and of low cost; it has a $95 \%$ sensitivity rate for extra-intestinal pathogenic infections. When results are negative and suspicion index is high, testing may be repeated at one week, to increase the negative predictive value. It is also useful for follow-up, because once treatment begins and medication is administered, negative results appear in 6 to 12 months (Anesi \& Gluckman).

\section{Morphological characteristics:}

a) Pathological anatomy: Macroscopic findings of AHA are, solid and well circumscribed cavities, initially filled with yellowish material, then brownish ("anchovy paste"); odorless and pasty (Fig. 4). The microscopic study shows a central area of fibrinoid necrosis with granulation tissue in the periphery, without neutrophils (not quite an abscess), with Entamoeba trophozoites of up to 60 microns with small eccentric nuclei and cytoplasmic vacuoles, that may contain phagocyte erythrocytes. Adjacent liver tissue presents with chronic inflammation, reactive hepatocytes and fibrosis (Anesi \& Gluckman).

b) Images (Tables I and II): US can provide information on a hypoechogenic lesion, with low intensity internal echoes and no significant wall echoes (Fig. 5A). Lesions tend to be singular and adopt a spherical or ovoid shape, with a 2 and 8 $\mathrm{cm}$ diameter. They usually present an incomplete edematous margin, with a smooth or nodular margin $(60$ and $40 \%$ of cases respectively) and internal septation (30\% of cases) (Garvin \& Willig, 2010). 
In the CT scan, well-defined lesions are observed, with attenuation values that indicate the presence of thick fluid (10-20 UH). A halo of peripheral edema and thickened wall is noted, with a thickness of 3-15 mm (Figs. 5B and 5C). When complicated by hepatobronchial or hepatocholic fistula, detritus level, septum and even gas may be noted (Wang \& Lin, 2018).

MRI is used in T1 to determine homogeneous hypointensity, in T2: homogeneous hyperintensity and perilesional edema in $50 \%$ of cases (Fig. 5D) (Garvin \& Willig).
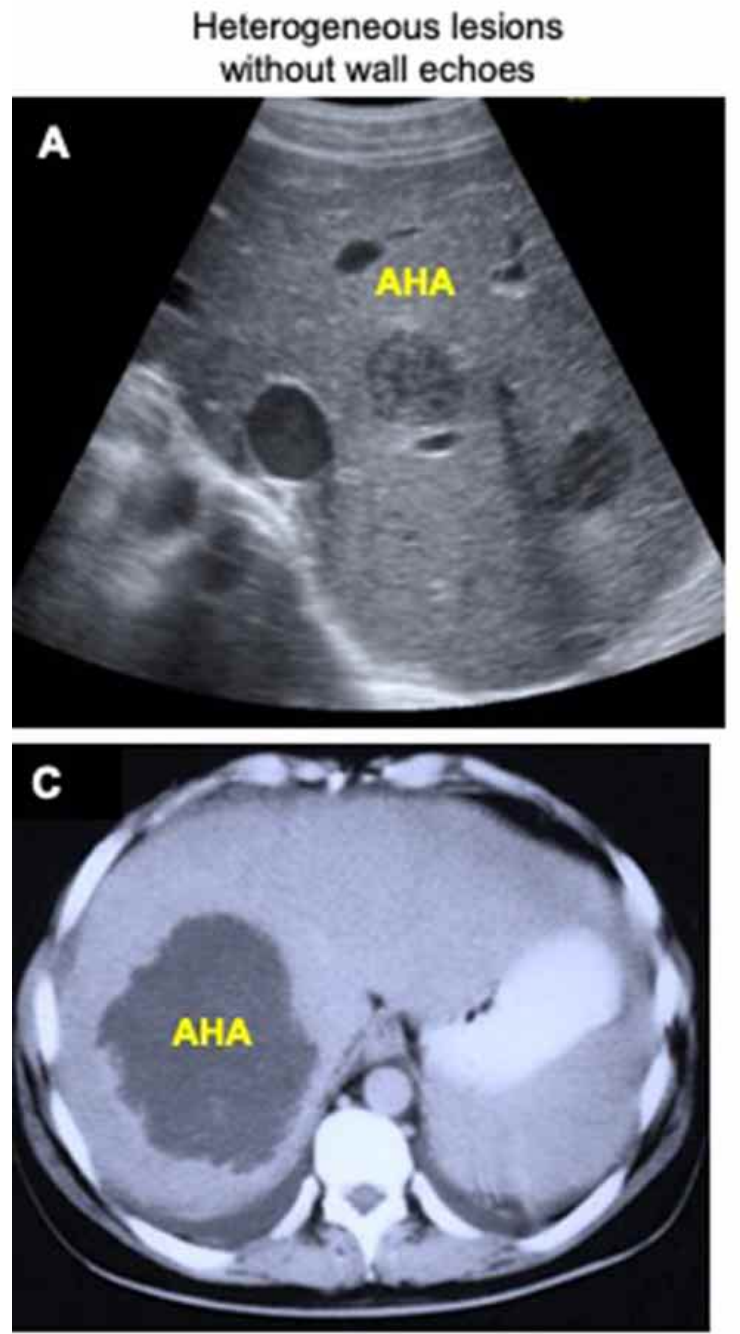

\section{Single hypointense lesion} with pseudocapsule
Radioisotope uptake (Tc-99m, Gallium) NM, allows confirmation of a "cold abscess", surrounded by a radioactivity margin (due to the absence of inside leukocytes) (Wang \& Lin).

In $75 \%$ to $96 \%$ of cases, serum Gal / GalNAc antigen by ELISA is positive. Following treatment onset it tends to become negative, it is therefore, useful to identify patients with acute symptoms, prior to the development of antibodies (Dinoop et al., 2016). PCR sensitivity to detect E. histolytica is known, and it is a useful diagnosis of non-pathogenic amoebas. However, it is expensive and is not standardized.
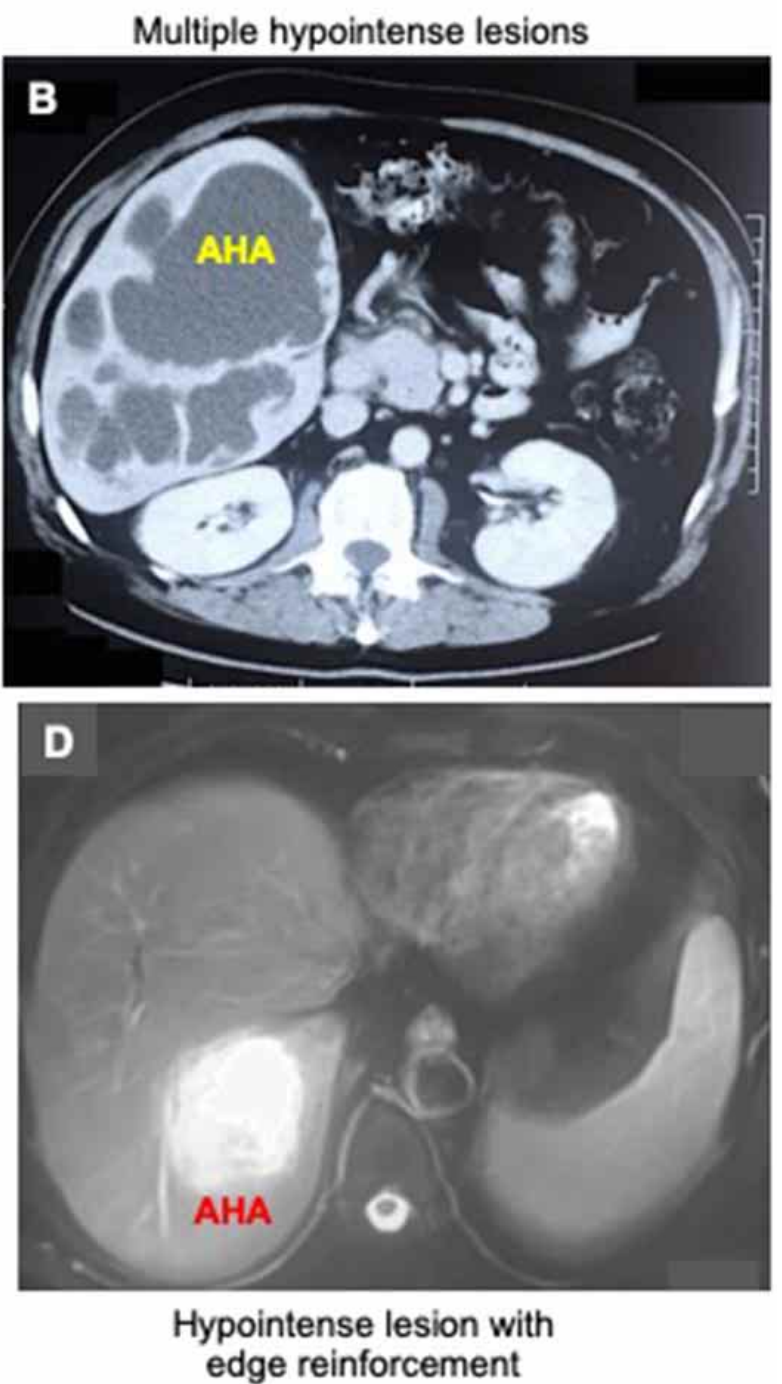

Fig. 5. A: Image of the US, in which the existence of heterogeneous hypoechogenic lesions without wall echoes, characteristics of AHA is verified. B: CT scan with contrast, in which multiple hypointense lesions surrounded by healthy parenchyma are observed. C: CT scan with contrast, in which a single hypointense lesion with pseudo capsule is identified, surrounded by apparently healthy parenchyma. D: NMR contrasted with Gadolinium. Hypointense lesion with margin reinforcement. 
Stool study is helpful in the diagnosis of intestinal disease. Such analyses are used to determine the likelihood of an existing AHA. They include serial examination of bowel movements for cysts and trophozoites, and even though it is reasonable to check for antigens in stool samples, in $60 \%$ of AHAs there is no coexistence with intestinal amebiasis (Anesi \& Gluckman; Lachish et al.).

Treatment: AHA treatment consists in the administration of amebicides, which in most uncomplicated cases is adequate. Once treatment with tissue factor amebicides to eliminate invasive trophozoites is completed, follow-up treatment with luminal action amebicides should be performed to eradicate intestinal colonization.

Reinfection has been reported in $10 \%$ of patients, on failure to administer the above follow-up treatment (Wang \& Lin).

Percutaneous image-guided drainage is recommended in the following cases: AHA larger than $5 \mathrm{~cm}$ in diameter, due to an increased risk of rupture; in cases located in the left liver, because they are associated with higher mortality rates, due to rupture to peritoneum or pericardium); and, when there is no response to medical treatment in 5 to 7 days, or there is concomitant liver failure. Surgical drainage is indicated when percutaneous drainage fails, the location is not accessible for the percutaneous technique, or during complications of the latter (Wang \& Lin).

\section{Hydatic hepatic abscess}

HHA is a developmental complication of Hepatic echinococcosis, once cyst integrity has been lost, which subsequently generates communication between cyst and bile ducts. Typically, it presents with nonspecific clinical manifestations, in contrast with the usual clinical course of abdominal abscesses. This may be explained by the presence of a pericystic membrane, which generates a nonspecific defense mechanism for the host. Hence, it is possible that clinical features and abnormal lab results, may not be the result of HHA. Results could however, be related to acute cholangitis, following migration of parasitic structures to the bile duct, or a secondary acute peritonitis when the cyst ruptures further, and into the peritoneum (Manterola et al., 2003, 2015; Bhutani \& Kajal, 2018; Shi et al.; Fadel et al., 2019).

The diagnosis is rarely considered, and not often suspected. Eventually, it is based on epidemiological background, clinical evidence and images (Karavias et al., 1988; Manterola et al., 2003).

\section{Morphological characteristics:}

a) Pathological anatomy: The macroscopic findings of AHH are as follows: Single cyst (75\% of cases), oval shaped, variable size (up to $20 \mathrm{~cm}$ in diameter); pearly white color, filled with purulent fluid and may contain gas (Figs. 6A and $6 B)$.

Microscopic studies allow confirmation of a 3-layer 10-25 microns thick wall (internal or germinal, with bacterial and polymorphonuclear elements; intermediate, $1 \mathrm{~mm}$ thick, avascular, eosinophilic, refractant and chitinous, stained strongly with PAS and GMS; and external, composed of fibrovascular tissue compacted by the growth of the cyst, with chronic inflammatory cells, with variable calcification), typical of a hydatid cyst (Karavias et al.; Bhutani \& Kajal).
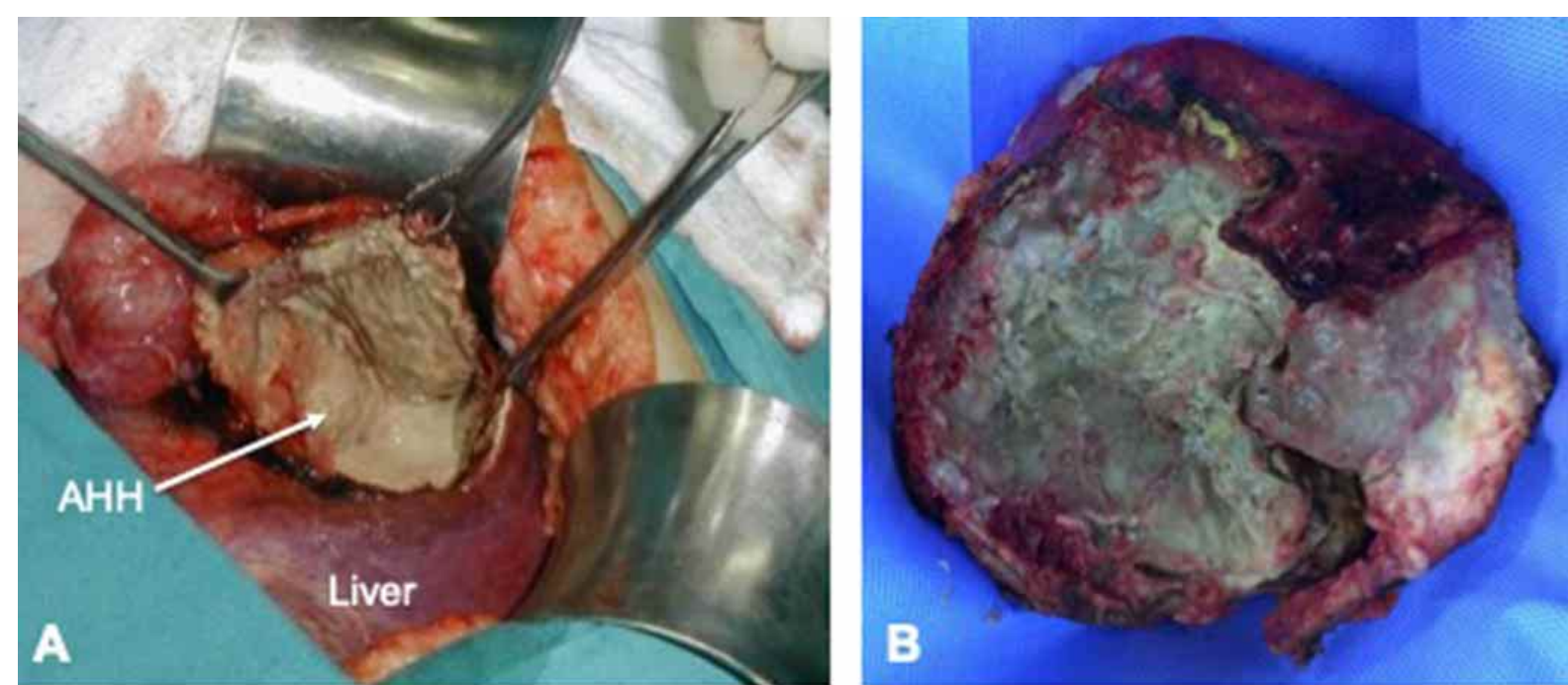

Fig. 6. A. Surgical image of an HHA. Open lesion is seen, with purulent material inside. B: Surgical piece, once resected (complete pericystic lesion). 
b) Images (Tables I and II): Conventional radiological findings (chest X-ray or simple abdomen) are: Elevation of the right hemi-diaphragm, subdiaphragmatic or intrahepatic hydro-air levels, curvilinear and ring calcification shadows (Karavias et al.; Salinas et al., 2000).

US is effective in identifying single or multiple cystic lesions (Fig. 7A). These may present with diverse aspects, echogenic material or septa (Salinas et al.). According to the WHO ultrasound findings classification (WHO Informal Working Group on Echinococcosis, 1996), there is evidence that in these cases CL type lesions can be detected (Manterola et al., 2003), CE 4 and CE 5 (Barosa et al., 2017; Alghofaily et al., 2017).

With the use of CT scan, cystic type lesions of variable fluid density, areas of peripheral focal calcification, septa and internal cysts can be identified (Fig. 7B). There may also be dilated intrahepatic bile ducts, the result of cyst compression or rupture (Manterola et al., 2015; Alghofaily et al.).

In the NMR, characteristics included in T1, hypointense mixed signal (depending on the amount of cellular material; $\mathrm{T} 2$, hyperintense mixed signal, there may be internal septa and cysts; and in T1 with Gadolinium: wall reinforcement and septa (Alghofaily et al.) (Fig. 7C).

Treatment: In the past, surgical drainage (cystostomy) was the treatment of choice which sometimes also included choledochostomy (bipolar drainage). However, evidence shows that cystostomy and bipolar drainage are associated with unsatisfactory postoperative evolution. Postoperative development of residual cavities, biliary fistulas and chronic suppuration have all been reported.
Such complications require further and more complex surgical interventions, possibly associated with increased morbidity and mortality. There is evidence of morbidity and mortality rates of $24.4 \%$ and $2 \%$, respectively (Salinas et al.; Manterola et al., 2015).

\section{DISCUSSION}

Even though HA morphology is variable, it follows specific patterns related to its etiology and dissemination. Current imaging techniques are reliable and allow diagnosis of the underlying cause. Furthermore, they are a useful guide for treatment and subsequent prognosis, as well as in differential diagnosis, with pathologies showing similar distribution patterns (primary neoplasms and liver metastases, hemangiomas, simple cysts, among others) (Abbas et al.; Lardière-Deguelte et al.; Akhondi \& Sabih).

High-performance imaging techniques (US, CAT, NMR and $\mathrm{NM}$ ), are proposed when hepatic infectious pathology is suspected, which should be determined based on their availability at hospitals or health care centers, as well as safety considering the patient's condition (Shi et al.). See Tables I and II.

In summary, HA is an infrequent and scarcely reported conditions. Depending largely on its heterogeneous distribution in the population, etiology, as well as the social and healthcare levels of the region under study. Considering all morphological characteristics, results may be favorable with treatment, depending on the etiology, size and location, thereby reducing the mortality rates inherent to the disease. Consequently, the analysis of this component through diagnostic imaging techniques is critical (Abbas et al.; Akhondi \& Sabih).

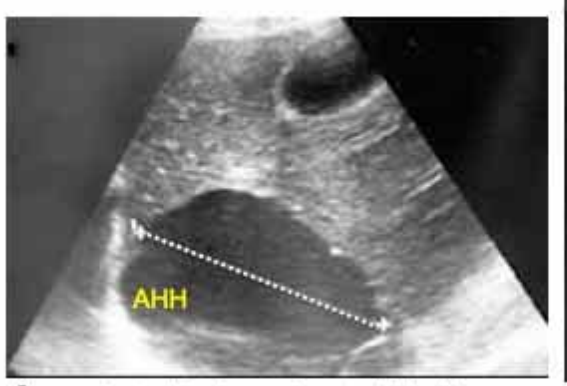

A Cyst with echogenic material inside

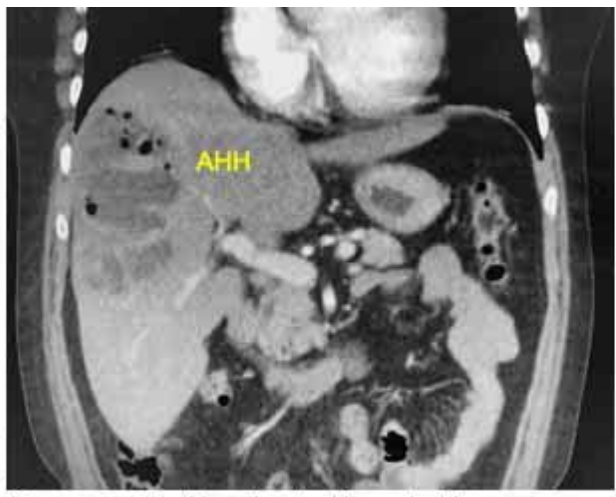

Partitioned cyst with gas inside

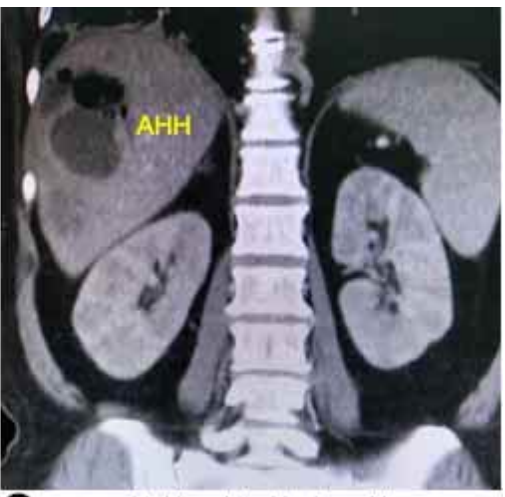

Multiloculated lesion with mixed hyperintensities in T2

Fig. 7. A. US of patient with HHA. A unique cystic lesion is identified, with echogenic material content. B. CT with contrast. There is a hypointense, rounded lesion with gas inside, typical of an HHA. C. NMR. Multiloculated lesion with mixed hyper intensities in T2 is observed. 
CASTillo, S. \& MANTEROLA, C. Características morfológicas de los abscesos hepáticos según su etiología. Int. J. Morphol., 38(2):406-414, 2020.

RESUMEN: Los abscesos hepáticos son entidades nosológicas de baja frecuencia, pero de significativa relevancia, la que se relacionada con las elevadas cifras de morbilidad y mortalidad que pueden causar si no son detectados y tratados a tiempo. Si bien se describen múltiples clasificaciones operacionales, aquella más reconocida por su utilidad es la relacionada a su agente causal (piógenos o bacterianos, hidatídicos o secundarios a hidatidosis infectada, y amebianos o por amebiasis invasora). El objetivo de este artículo fue generar un documento de estudio respecto de las características morfológicas de los abscesos hepáticos, caracterizándolos de acuerdo con su etiología, así como describir su estudio y tratamiento recomendado de acuerdo con el estado del arte.

PALABRAS CLAVE: Absceso hepático; Absceso hepático hidatídico; Absceso hepáticoamebiano; Absceso hepático piógeno.

\section{REFERENCES}

Abbas, M. T.; Khan, F. Y.; Muhsin, S. A.; Al-Dehwe, B.; Abukamar, M. \& Elzouki, A. N. Epidemiology, clinical features and outcome of liver abscess: a single reference center experience in Qatar. Oman Med. J., 29(4):260-3, 2014.

Akhondi, H. \& Sabih, D. E. Liver Abscess. StatPearls website. Treasure Island (FL), StatPearls Publishing, 2019.

Alam, F.; Salam, M. A.; Hassan, P.; Mahmood, I.; Kabir, M. \& Haque, R. Amebic liver abscess in Northern Region of Bangladesh: sociodemographic determinants and clinical outcomes. BMC Res. Notes, 7:625, 2014.

Alghofaily, K. A.; Saeedan, M. B.; Aljohani, I. M.; Alrasheed, M.; McWilliams, S.; Aldosary, A. \& Neimatallah, M. Hepatic hydatid disease complications: review of imaging findings and clinical implications. Abdom. Radiol. (N. Y.), 42(1):199-210, 2017.

Anesi, J. A.; \& Gluckman, S. Amebic liver abscess. Clin. Liver Dis. (Hoboken), 6(2):41-3, 2015.

Barosa, R.; Pinto, J.; Caldeira, A. \& Pereira, E. Modern role of clinical ultrasound in liver abscess and echinococcosis. J. Med. Ultrason. (2001), 44(3):239-45, 2017.

Bhutani, N. \& Kajal, P. Hepatic echinococcosis: A review. Ann. Med. Surg. (Lond.), 36:99-105, 2018.

Dinoop, K. P.; Parija, S. C.; Mandal, J.; Swaminathan, R. P. \& Narayanan, P. Comparison of nested-multiplex, Taqman \& SYBR Green real-time PCR in diagnosis of amoebic liver abscess in a tertiary health care institute in India. Indian J. Med. Res., 143(1):49-56, 2016.

Fadel, S. A.; Asmar, K.; Faraj, W.; Khalife, M.; Haddad, M. \& El-Merhi, F. Clinical review of liver hydatid disease and its unusual presentations in developing countries. Abdom. Radiol. (N. Y.), 44(4):1331-9, 2019.

Garvin, K. W. \& Willig, J. H. Amebic liver abscess. Am. J. Trop. Med. Hyg., 83(5):961, 2010.

González-Alcaide, G.; Peris, J. \& Ramos, J. M. Areas of research and clinical approaches to the study of liver abscess. World J. Gastroenterol., 23(2):357-65, 2017.

Karavias, D.; Panagopoulos, C.; Vagianos, C.; Vagenas, C.; Rathosis, S. \& Androulakis, J. Infected echinococcal cyst. A common cause of pyogenic hepatic abscess. Ups. J. Med. Sci., 93(3):289-96, 1988.
Lachish, T.; Wieder-Finesod, A. \& Schwartz, E. Amebic Liver abscess in israeli travelers: a retrospective study. Am. J. Trop. Med. Hyg., 94(5):1015-9, 2016.

Lardière-Deguelte, S.; Ragot, E.; Amroun, K.; Piardi, T.; Dokmak, S.; Bruno, O.; Appere, F.; Sibert, A.; Hoeffel, C.; Sommacale, D.; et al. Hepatic abscess: diagnosis and management. J. Visc. Surg., 152(4):231-43, 2015.

Longworth, S. \& Han, J. Pyogenic liver abscess. Clin. Liver Dis. (Hoboken), $6(2): 51-4,2015$.

Lübbert, C.; Wiegand, J. \& Karlas, T. Therapy of Liver Abscesses. Viszeralmedizin, 30(5):334-41, 2014.

Manterola, C.; Barroso, M.; Vial, M.; Bustos, L.; Muñoz, S.; Losada, H.; Bello, N.; Hernández, F. \& Carrasco, R. Liver abscess of hydatid origin: clinical features and results of aggressive treatment. A. N. Z. J. Surg., 73(4):220-4, 2003.

Manterola, C.; Urrutia, S. \& MINCIR GROUP. Infected hepatic echinococcosis: results of surgical treatment of a consecutive series of patients. Surg. Infect. (Larchmt.), 16(5):553-7, 2015.

Mischnik, A.; Kern, W. V. \& Thimme, R. Pyogenic liver abscess: changes of organisms and consequences for diagnosis and therapy. Dtsch. Med. Wochenschr., 142(14):1067-74, 2017.

Pinilla, A. E.; López, M. C.; Castillo, B.; Murcia, M. I.; Nicholls, R. S.; Duque, S. \& Orozco, L. C. A diagnostic approach to hepatic abscess. Rev. Med. Chil., 131(12):1411-20, 2003.

Piqueras, A. I.; Macia, M.; Ibañez, L. \& Esteban, M. J. Pyogenic liver abscess with delayed presentation after appendectomy. Pediatr. Infect. Dis. J., 35(7):817-9, 2016.

Salinas, J. C.; Torcal, J.; Lozano, R.; Sousa, R.; Morandeira, A. \& Cabezali, R. Intracystic infection of liver hydatidosis. Hepatogastroenterology., 47(34):1052-5, 2000.

Shi, S. H.; Zhai, Z. L. \& Zheng, S. S. Pyogenic liver abscess of biliary origin: the existing problems and their strategies. Semin. Liver Dis., 38(3):270-83, 2018

Wang, H. H. \& Lin, W. R. Amebic liver abscess. N. Engl. J. Med., 379(23):2255, 2018.

WHO Informal Working Group on Echinococcosis. Guidelines for Treatment of Cystic and Alveolar Echinococcosis in Humans. WHO Informal Working Group on Echinococcosis. Bull. World Health Organ., 74(3):231-42, 1996

Zibari, G. B.; Maguire, S.; Aultman, D. F.; McMillan, R. W. \& McDonald, J. C. Pyogenic liver abscess. Surg. Infect. (Larchmt.), 1(1):15-21, 2000.

Corresponding author:

Dr. Carlos Manterola

Department of Surgery \& CEMyQ

Universidad de La Frontera

Temuco

CHILE

E-mail: carlos.manterola@ufrontera.cl

Received: 01-10-2019

Accepted: $28-11-2019$ 